\title{
Development of a Low-Cost Sensing Node with Active Ven- tilation Fan for Air Pollution Monitoring
}

\author{
Nicolas P. Winkler ${ }^{1}$, Patrick P. Neumann ${ }^{1}$, Harald Kohlhoff', Jessica Erdmann ${ }^{1}$, Erik Schaffernicht ${ }^{2}$, \\ Achim J. Lilientha/ ${ }^{2}$ \\ ${ }^{1}$ Bundesanstalt für Materialforschung und -prüfung, Unter den Eichen 87, 12205 Berlin, Germany \\ 2 Örebro University, SE-701 82 Örebro, Sweden \\ Contact e-mail address: mro@bam.de
}

\begin{abstract}
Summary:
A fully designed low-cost sensing node for air pollution monitoring and calibration results for several lowcost gas sensors are presented. As the state of the art is lacking information on the importance of an active ventilation system, the effect of an active fan is compared to the passive ventilation of a lamellar structured casing. Measurements obtained in an urban outdoor environment show that readings of the low-cost dust sensor (Sharp GP2Y1010AU0F) are distorted by the active ventilation system. While this behavior requires further research, a correlation with temperature and humidity inside the node shown.
\end{abstract}

Keywords: wireless sensing node, low-cost, air pollution, sensor network, occupational health

\section{Background and Motivation}

Air pollution is the biggest environmental health risk in the European Union (EU). It is estimated that 400,000 premature deaths are caused by air pollutants per year. The EU aims to reduce this number by $52 \%$ by 2030 compared to 2005 [1]. Especially in industrial environments, where workers are exposed to airborne by-products, continuous monitoring is crucial to develop and comply with occupational health standards.

Rapid developments in sensor technology enable new and cost-efficient possibilities for longterm monitoring of air parameters. A concept that combines the advantages of stationary low-cost and mobile high-quality sensors in one system was proposed in [2] and is the objective of the research project "Robot-assisted Environmental Monitoring for Air Quality Assessment in Industrial Scenarios" (RASEM).

\section{Related Work}

Although many approaches and projects aim to leverage low-cost devices for a next-generation air pollution monitoring system, research shows that state-of-the-art devices cannot replace traditional reference monitoring stations, because they lack accuracy and require extensive calibration in laboratory and field [3][4][5].

Hardware platforms used by other researchers were analyzed and compared in [6]. An Arduino microcontroller with similar low-cost sensors used in this project has been used for dense pollution monitoring in foundries [7]. While previous research papers focus on the performance of specific sensors (e.g., [4]) or ready-to-buy- solutions (e.g., [3]), there is little information on the overall manufactured design (e.g., casing) or how important an active ventilation system is.

\section{Sensing Node Setup}

Compared to the state of the art, this sensing node includes a fan for active ventilation. The sensors are sheltered by a commercially available weather protective casing, which enables passive ventilation through its lamellar structure.

The Arduino framework is used, which comes with a high variety of available software libraries. An ESP32 microcontroller (Espressif ESP32DevKitC-32U), which is compatible with Arduino, a real-time clock (Adafruit DS3231), and a micro SD breakout board (Adafruit MicroSD) are housed in a 3D-printed casing at the bottom of the sensing node. Data is transmitted via antenna and WLAN to a central server unit.
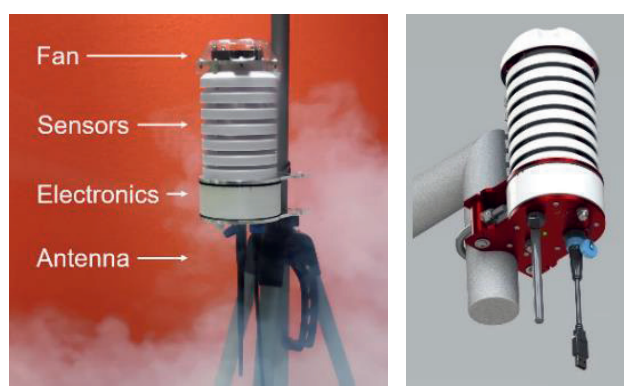

Fig. 1: Left: Sensing node during tests with a fog machine. Right: 3D rendering of a sensing node.

\section{Environmental Sensors}

\section{A. DHT22 Temperature \& Humidity Sensor}

An Adafruit AM2302 is used, which is a wired DHT22 temperature and humidity sensor. 
According to the datasheet, operating ranges (and accuracies) are -40 to $80{ }^{\circ} \mathrm{C}\left( \pm 0.5^{\circ} \mathrm{C}\right)$ for temperature and 0 to $100 \%( \pm 2 \%)$ for relative humidity, however [4] indicates that the error for humidity readings is up to $5 \%$.

\section{B. WaveShare Dust Sensor}

This WaveShare dust sensor board is a Sharp GP2Y1010AU0F optical dust sensor with resistors on board. The manufacturer specifies the measurement range with up to $500 \mu \mathrm{g} / \mathrm{m}^{3}$, however, previous research by [5] shows that the sensor signal outputs are not well correlated to the mass concentration. To adapt the sensor board to the ESP32's analog-to-digital converter, the existing resistors are changed to increase the maximum sensor output to $1.1 \mathrm{~V}$.

\section{SPEC Digital Gas Sensor}

The digital gas sensor module by SPEC Sensors can be used with different electrochemical sensors. $8 \mathrm{CO}$ and 16 Indoor Air Quality (IAQ, COcalibrated) are three-point calibrated with dry reference gases at room temperature (Fig. 2).

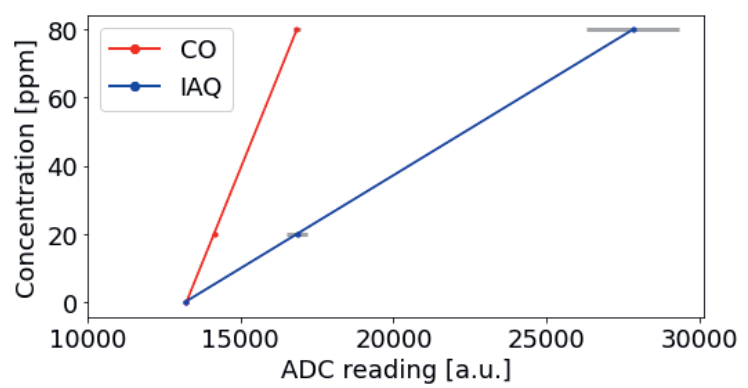

Fig. 2: Three-point calibration results with standard deviation $\sigma$ and fitted function $a x+b$ (CO: $a=0.0222$, $b=-293.8$; IAQ: $a=0.0055, b=-72.3$ ).

\section{Influence of Active Ventilation}

All sensors are sampled directly before and after a two-second runtime of the fan (measurement period $=30 \mathrm{~s}$ ). Fig. 3 shows the dust sensor readings of three consecutive days in an urban outdoor environment in Berlin-Lichterfelde in early June 2020 . The sensor readings taken directly after the fan runtime suffer from noticeably high variance. This effect correlates with the temperature and humidity measurements inside the node. According to external weather data, the first day was cloudy, whereas the following two days were mostly sunny. Probably, the sun heats the casing, which leads to distorted sensor measurements. This is also indicated by the recurrent drop in temperature in the afternoon, which is most likely caused by the neighboring building shadowing the sensing node. The calculated global Pearson correlation coefficient $r$ is $r=0.28$ (dust and temperature), $r=0.012$ (dust and humidity) and $r=-0.79$ (temperature and humidity). An influence of the fan on the other sensors is not visible.

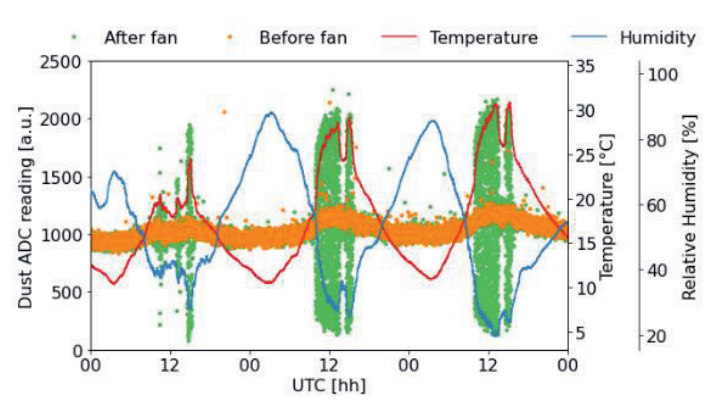

Fig. 3: Three days of dust and temperature sensor readings. Variance in dust samplings correlates with the temperature inside the sensing node.

\section{Conclusion}

More investigations are needed to explain and verify the influence of an active fan on dust sensor readings. Future experiments will investigate how this effect diminishes after turning off the fan. Apart from that, further research may identify how low-cost sensors can support traditional reference sensors for environmental monitoring.

\section{Acknowledgment}

The authors express their gratitude to SAFERA for funding the project in the scope of the 2018 joint call and thank the involved colleagues from the Finnish Institute for Occupational Health.

\section{References}

[1] European Union, A Clean Air Programme for Europe (COM(2013) 918 final), (2013);

[2] N.P. Winkler, P.P. Neumann, A. Säämänen, E. Schaffernicht, A.J. Lilienthal, High-quality meets lowcost: Approaches for hybrid-mobility sensor networks, Materials Today: Proceedings 32, 250-253 (2020); doi: 10.1016/j.matpr.2020.05.799

[3] N. Castell et al., Can commercial low-cost sensor platforms contribute to air quality monitoring and exposure estimates?, Environment International 99, 293-302 (2017); doi: 10.1016/j.envint.2016.12.007.

[4] T. Araújo, L. Silva, A. Moreira, Evaluation of LowCost Sensors for Weather and Carbon Dioxide Monitoring in Internet of Things Context, loT 1, 286-308 (2020), doi: 10.3390/iot1020017

[5] J. Li, P. Biswas, Optical Characterization Studies of a Low-Cost Particle Sensor, Aerosol and Air Quality Research 17 7, 1691-1704 (2017); doi: 10.4209/aaqr.2017.02.0085

[6] F. Karray, M.W. Jmal, A. Garcia-Ortiz, M. Abid, A.M. Obeid, A comprehensive survey on wireless sensor node hardware platforms, Computer Networks 144 , 89-110 (2018), doi: 10.1016/j.comnet.2018.05.010

[7] V.H. Bennetts et al., Towards occupational health improvement in foundries through dense dust and pollution monitoring using a complementary approach with mobile and stationary sensing nodes, 2016 IEEE/RSJ International Conference on Intelligent Robots and Systems (IROS), 131-36 (2016); doi: 10.1109/IROS.2016.7759045 